\title{
Critical state constitutive models and shear loading of overconsolidated clays with deviatoric hardening
}

https://doi.org/10.2478/sgem-2019-0024

received May 20, 2019; accepted July 11, 2019.

\begin{abstract}
This paper presents an enhanced constitutive model integrating deviatoric hardening with a modified yield surface for overconsolidated clayey soils in a general framework of Cam-clay type models. Its performance was assessed with the simulation of drained and undrained triaxial tests on three clays at different consolidation states in comparison to two critical state models. The proposed model satisfactorily estimates the shear resistance, while capturing the smooth nonlinearity of the soil response.

Shear triaxial tests at constant mean pressure were performed on an overconsolidated marl to study the shear response. Their simulation attests the importance of deviatoric hardening integration.
\end{abstract}

Keywords: constitutive models, critical state, overconsolidated clay, shear loading, deviatoric hardening.

\section{Introduction}

Many geotechnical applications, such as retaining walls and tunneling, take place in overconsolidated (OC) clays and involve the mobilization of the soil's shear resistance. The quality of finite element simulations closely depends on the choice of the soil constitutive model. However, all the characteristic behaviors of geomaterials cannot be reproduced by one existing model under different loading conditions with the assumptions of a particular theory. An appropriate soil model for OC clays is then required for simulating their characteristic softening and

\footnotetext{
*Corresponding author: Sara Rachdi, PhD student, Centre de Géosciences, MINES ParisTech, 35 rue Saint-Honoré, 77300 , Fontainebleau France; e-mail: sara.rachdi@mines-paristech.fr Emad Jahangir, Michel Tijani, PhD, Centre de Géosciences, MINES ParisTech, 35 rue Saint-Honoré, 77300 , Fontainebleau France Jean-François Serratrice, PhD, Cerema Méditerranée, 30 rue Albert Einstein, CS 70499, 13593 Aix en Provence France
}

dilating response with a realistic estimation of their shear resistance. This work focuses on the enhancement of the simulation of OC clay behavior at the sample level.

Clayey soil behavior is best simulated in the framework of critical state theory that was featured by Roscoe, Schofield, and Wroth (1958) based on an exhaustive triaxial test campaign. Several enhancements were introduced to this theory such as the consideration of fabric effects (Dafalias, 2016) or the use of nonlinear formulas for the critical state line (e.g., Jin, Xu, \& Hicher, 2017). The search of a suitable constitutive model for OC clays, in the framework of critical state theory is still a topical issue (Y. Chen \& Yang, 2017).

The modified Cam-clay (MCC) model was introduced by Roscoe and Burland (1968) as the prototype of soil models in this context. It has been the subject of several extensions such as the incorporation of the influence of the soil structure and destructuration (e.g., Liu \& Carter, 2002; Suebsuk, Horpibulsuk, \& Liu, 2010, 2011) or the integration of the anisotropy of plasticity using rotational hardening (e.g., Dafalias, 1986; Dafalias, Manzari, \& Papadimitriou, 2006; Dafalias \& Taiebat, 2013).

A great number of models were proposed based on the MCC model to overcome some of its drawbacks, as reviewed in the work of Gens and Potts (1988) such as excessive dilatancy (Lagioia \& Potts, 1988; Potts \& Zdravkovic, 1999), the overestimation of the shear resistance of OC clays and the sharp transition from elasticity to plasticity. Bounding surface models (e.g., Mroz \& Zienkiewicz, 1984; Dafalias, 1986) and inner yield surface models (e.g., Chakraborty, Salgado, \& Loukidis, 2013; Hong, Pereira, Tang, \& Cui, 2016) presented a solution to the latest point. However, such models are still not commonly used in engineering practice because of their complexity. A realistic estimation of the shear resistance of OC clays can be improved by modifying the MCC model formulation on the dry side (e.g., J. Chen, 2017) or by introducing a modified yield surface as in the clay and sand model (CASM) (Yu, 1998) that corrects the overestimation of the peak resistance and the prediction of dilatation. Early-stage dilatation of OC clays was studied by Hattab and Hicher (2004) 
through a series of pure shear tests. They suggested that an independent deviatoric mechanism is necessary.

In practice, other than the classic Mohr-Coulomb model, the hardening soil model (HSM) (Schanz \& Vermeer, 2000) is one of the most popular models used in geotechnical structure design in France, especially for the Grand Paris Express Project (Gilelron, 2016). In spite of some reported drawbacks of the HSM (e.g., Obrzud, 2010; Truty \& Obrzud, 2015), the integration of both deviatoric and volumetric hardening mechanisms with a different loading and unloading modulus has earned it wide renown in geotechnical engineering practice. However, it is only adapted for normally consolidated soils. It does not include the critical state concept and omits the simulation of soil softening. More generalist formulations of constitutive models were presented as the hierarchical single surface (HISS) modeling approach that allows the development of models of different levels of complexities to take into account many soil characteristics as anisotropy and damage mechanisms (C. S. Desai, 1980; C. Desai, Somasundaram, \& Frantziskonis, 1986). However, it is not used in engineering practice.

In an attempt to close the gap between the sophisticated academic models and the models used in engineering practice, the aim of this paper is to present a critical state soil constitutive model, with deviatoric and volumetric hardening mechanisms, adapted to simulate the behavior of OC clays under shear loading. Critical state theory presents a framework broadly used for the development of several soil models that could be classified as Cam-clay type models. Many of these models can be grouped into a general formalism that makes their comparison easier. The proposed model will be compared not only to the MCC model but also to CASM, which uses a non-associative flow rule and was validated for both normally consolidated and overconsolidated clays. To understand the coupling between shear and volumetric hardening mechanisms, a series of specific triaxial tests with controlled stress paths were conducted on a natural overconsolidated gray marl.

This paper is organized as follows. A general framework for the formulation of Cam-clay type models is presented in Section 2. The formulation of the MCC model and CASM is presented explicitly prior to the presentation of the proposed SCSM. In Section 3, the three models are fitted on experimental data from undrained (3.1) and drained triaxial tests (3.2) at different consolidation states and from shear loading tests conducted at constant mean pressure (3.3). The results of these tests at different stress levels highlight the importance of deviatoric hardening in critical state models.

\section{A soil constitutive model family}

The soil thermodynamic state is characterized by the Cauchy effective stress tensor $\underline{\underline{\sigma}}$ (negative in compression) and a list $\alpha$ of scalar hardening variables. If the soil is isotropic and the Lode angle is not used in the constitutive model equations, the state function depends only on $p, q$ and $\alpha$, where $p$ and $q$ stand for the volumetric and deviatoric parts, respectively, of the stress tensor defined as follows.

$$
\begin{gathered}
p=-\frac{\operatorname{tr}(\underline{\underline{\sigma}})}{3} \\
\underline{\underline{s}}=\underline{\underline{\sigma}}+p \underline{\underline{1}} \\
q=\sqrt{\frac{3}{2}}\|\underline{\underline{s}}\| \text { where }\|\underline{\underline{s}}\|=\sqrt{\underline{\underline{s}}: \underline{\underline{s}}} \\
n=\underline{\underline{s}} /\|\underline{\underline{s}}\| \text { if } q>0
\end{gathered}
$$

In the theory of elastoplasticity, the total strain rate is decomposed into elastic and plastic components (partition law).

$$
\underline{\underline{\varepsilon}}=\underline{\dot{\varepsilon}}^{e}+\underline{\dot{\varepsilon}}^{p}
$$

The rates such as $\underline{\dot{\varepsilon}}=d \underline{\varepsilon} / d t$ use a kinematic time $t$ (as any strictly increasing function of actual time).

It is acknowledged that volume changes are an important feature of the mechanical soil response. On the other hand, undrained loading entails pure shearing at constant-volume deformation. It is then suitable to divide the strain rate tensor into volumetric and deviatoric components. Particularly, the plastic strain rate tensor is written as follows:

$$
\underline{\underline{\dot{\varepsilon}}}^{p}=\left(\frac{\dot{\zeta}}{3}\right) \underline{\underline{\mathbf{1}}}+\underline{\underline{\dot{\varepsilon}}}^{p^{\prime}}
$$

where $\zeta=\operatorname{tr}\left(\underline{\varepsilon}^{p}\right)$ is the volumetric plastic strain and $\underline{\underline{\varepsilon}}^{p}$ is the deviatoric plastic strain tensor. ${ }^{1}$

In the general framework of elastoplasticity, the evolution of the plastic volumetric strain is written $\underline{\underline{\dot{\varepsilon}}}^{p}=\dot{\grave{\lambda}} \underline{\underline{a}}$

1 For any tensor $\underline{\beta}$, its deviatoric part is designated by a prime: $\underline{\beta}^{\prime}=\underline{\beta}-\frac{1}{3} \operatorname{tr}(\underline{\underline{\beta}}) \underline{\underline{1}}$, where $\underline{\underline{1}}$ is the identity tensor and $\operatorname{tr}(-)$ denotes the trace operator. 
where $\dot{\lambda} \geq \mathbf{0}$ is the plastic multiplier and a is a function of the state $(\underline{\underline{\sigma}}, \boldsymbol{\alpha})$. It is then deduced that $\underline{\underline{\dot{\varepsilon}}}^{\overline{\bar{p}}}=\dot{\boldsymbol{\lambda}} \underline{\underline{\boldsymbol{\alpha}^{\prime}}}$.

In this work, only materials such $\overline{\bar{a}} \underline{\underline{\boldsymbol{\alpha}}}^{\prime}=\underline{\overline{\mathbf{0}}}$ for $\boldsymbol{q}=\mathbf{0}$ and $\underline{\underline{\boldsymbol{a}}}^{\prime}=\underline{\underline{\boldsymbol{n}}}$ when $\boldsymbol{q}>\mathbf{0}$ are considered. Since the studied soils are isotropic and the hardening variables are scalars, this restriction ( $\underline{\underline{\boldsymbol{a}}}^{\prime}$ parallel to $\underline{\underline{\boldsymbol{n}}}$ traduces the fact that the plastic potential is independent of the Lode angle. Let us introduce the classic cumulative variable $\gamma$ (plastic shear strain), the rate of which is $\dot{\gamma}=\sqrt{\frac{2}{3}}\left\|\underline{\underline{\dot{p}^{\prime}}}\right\|$ and assume that $\dot{\gamma}=\mathbf{0}$ when $\boldsymbol{q}=\mathbf{0}$. Therefore, $\underline{\underline{\dot{\varepsilon}}}^{\prime}=\dot{\boldsymbol{\gamma}} \underline{\underline{\boldsymbol{n}}}$ and $\dot{\lambda}=\dot{\boldsymbol{\gamma}}$.

The list of hardening variables $\alpha$ is restricted to the scalars $\zeta$ and $\gamma$ for the studied models in this work.

\subsection{Isotropic compression}

The focus will first be on isotropic compression modeling. In Cam-clay type models, the change in the volume of a soil skeleton with the mean effective stress $p$ is governed by the double linearity of the specific volume $v=\mathbf{1}+\boldsymbol{e}$ (where $\boldsymbol{e}$ is the void ratio) and the logarithm of the effective pressure $\ln \boldsymbol{p}$ curves $(\boldsymbol{v} ; \boldsymbol{l n} \boldsymbol{p})$. This is illustrated in Figure 1 as an idealization of the experimentally observed behavior. With the hypothesis of small strains, the change in volumetric strain $\varepsilon_{v}$ is related to the change in the specific volume as $\operatorname{tr}(\underline{\dot{\varepsilon}})=-\frac{\dot{v}}{\dot{v}_{0}}$.

As a consequence, the volumetric strain rate is given as a function of the initial void ratio, the slope of the swelling line $\boldsymbol{\kappa}$ and the slope of the normal consolidation line $\lambda$. The elastic volumetric strain will be denoted as $\zeta$.

$$
\begin{gathered}
\dot{\zeta}^{e}=-\boldsymbol{\kappa}^{*} \frac{\dot{\boldsymbol{p}}}{\boldsymbol{p}} \\
\dot{\zeta}=-\left(\lambda^{*}-\boldsymbol{\kappa}^{*}\right) \frac{\dot{p}_{c}}{\boldsymbol{p}_{c}}
\end{gathered}
$$

where $\boldsymbol{\kappa}^{*}=\frac{\kappa}{1+e}$ and $\lambda^{*}=\frac{\lambda}{1+e}$.

Equation (8) can be rewritten to give the volumetric hardening law or the evolution of the preconsolidation pressure with the plastic volumetric strain.

$$
\boldsymbol{p}_{c}=\boldsymbol{p}_{c 0} \exp \left(-\frac{\zeta}{\lambda^{*}-\boldsymbol{\kappa}^{*}}\right)
$$

The studied constitutive models in this paper include the elastic behavior of the MCC model in addition to the volumetric behavior based on the double linearity of the $(v ; \ln \boldsymbol{p})$ curves as described above.

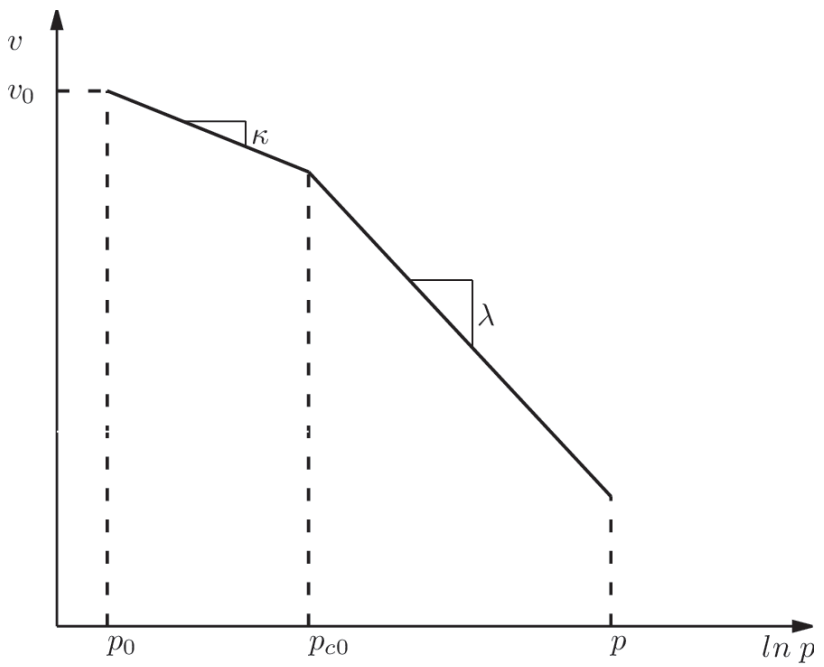

Figure 1: Schematic representation of the compressibility of a soil.

\subsection{Elastic behavior}

The studied models adopt the pressure-dependent elasticity of the MCC model with a constant Poisson's ratio v. Elastic strains are given by Equation (10).

$$
\underline{\dot{\varepsilon}}^{e}=\frac{\boldsymbol{\kappa}^{*}}{3 p}\left(-\dot{p} \underline{\underline{1}}+\frac{1+v}{1-2 v} \underline{\dot{s}}\right)
$$

The isotropic unloading and reloading are supposed to be elastic. This formulation for elastic behavior does not satisfy the principle of energy conservation for elastic loading as shown by Zytynski, Randolph, and Wroth (1978) and discussed by Wood (2003). For thermodynamic consistency during cyclic loadings, an alternative is a constant shear modulus $G$ independent of the stress level. A pressure dependent energy conservative elasticity can be derived from a hyperelastic energy potential function as presented by Einav and Puzrin (2004) and Houlbsy, Amorosi, and Rojas (2005). For monotonic loading, the elastic behavior of the MCC model is accepted and widely used in popular soil models.

\subsection{Generalized yield criterion and flow rule}

In stress space, the region of elastic behavior is defined by a yield criterion as a function of the stress tensor $\underline{\sigma}$ and a list $\alpha$ of scalar hardening variables $\boldsymbol{f}(\underline{\underline{\sigma}}, \boldsymbol{\alpha})$. For this family of soil models, for $\mathbf{0}<\boldsymbol{p}<\boldsymbol{p}_{\boldsymbol{c}}(\zeta)$, plastic strains are zero as long as $\boldsymbol{f}(\underline{\underline{\sigma}}, \boldsymbol{\alpha})<\mathbf{0}$ . The yield criterion is then written by Equations (1F) and 
(12). The preconsolidation pressure $\boldsymbol{p}_{c}$ evolves with the plastic volumetric strain $\zeta$, which is a common hardening variable for these models, according to Equation (8).

$$
\begin{gathered}
0<\boldsymbol{p}<\boldsymbol{p}_{c}(\zeta) \\
f(\underline{\underline{\sigma}}, \alpha)=q-\phi(p, \alpha)<0
\end{gathered}
$$

For an isotropic loading $(\boldsymbol{q}=\boldsymbol{0})$, the soil response is controlled by Equation (11) for the yield criterion and Equation (9) for the hardening rule.

For any other loading path, the yield criterion is defined by both Inequalities (11) and (12). When $f(\underline{\underline{\sigma}}, \boldsymbol{\alpha})=\mathbf{0}, \boldsymbol{q}=\boldsymbol{\phi}(\underline{\underline{\sigma}}, \boldsymbol{\alpha})>\mathbf{0}$ and $\gamma>0$.

This parameter $\boldsymbol{\gamma}$ is used as a plastic multiplier (as explained in the end of the introductory part of Section 2) and plastic strain rate tensor is written as follows.

$$
\underline{\underline{\dot{\varepsilon}}}^{p}=\dot{\gamma}\left[\frac{1}{3} \dot{\underline{\gamma}} \underline{\underline{1}}+\sqrt{\frac{3}{2}} \underline{\underline{n}}\right]
$$

The evolution of the plastic strains is fully determined with a function $\boldsymbol{\psi}(\boldsymbol{\eta}, \boldsymbol{\alpha})$, where $\boldsymbol{\eta}=\frac{q}{p}$ such as; $\frac{\dot{\xi}}{\gamma}=\psi(\boldsymbol{\eta}, \boldsymbol{\alpha})$.

In some soil models, the arguments of $\boldsymbol{\psi}$ are $(\boldsymbol{p}, \boldsymbol{\alpha})$ as are those of $\boldsymbol{\phi}$, which is no problem since $\psi$ is needed only when $\boldsymbol{q}=\boldsymbol{\phi}(\boldsymbol{p}, \boldsymbol{\alpha})$. The function $\boldsymbol{\phi}$ is such that the relationship between $\boldsymbol{\eta}$ and $\boldsymbol{p}$ for each $\boldsymbol{\alpha}$ is bijective.

Therefore, there is a function $\Phi$ such that $\boldsymbol{\eta}=\frac{\phi(p, \boldsymbol{\alpha})}{p}$ is equivalent to $\boldsymbol{p}=\boldsymbol{\Phi}(\boldsymbol{\eta}, \boldsymbol{\alpha})$. As a result, all these models have a flow rule that is derived from a plastic potential $g(\underline{\underline{\sigma}}, \boldsymbol{\alpha})=\boldsymbol{q}-\overline{\boldsymbol{\phi}}(\boldsymbol{p}, \boldsymbol{\alpha})$ where $\bar{\phi}(p, \alpha)=\int_{p_{0}}^{p} \psi\left(\frac{\phi(x, \alpha)}{x}, \alpha\right) d x$.

Conversely, from a given plastic potential, $\boldsymbol{\psi}(\boldsymbol{\eta}, \boldsymbol{\alpha})=\left(\partial_{p} \bar{\phi}\right)(\boldsymbol{\Phi}(\boldsymbol{\eta}, \boldsymbol{\alpha}), \boldsymbol{\alpha})$.

This last comment does not go beyond curiosity since a soil constitutive model can be formulated in the framework of elastoplasticity with a flow rule that is not derived from a plastic potential (Tijani, 2008). The restrictions on the flow rule come from thermodynamic considerations expressed by the Clausius-Duhem inequality, which states that the intrinsic dissipation must be positive. This inequality is written in terms of the plastic power among others. From Equation (13), the expression of the plastic power per unit of volume is $\boldsymbol{\varpi}=\underline{\underline{\sigma}}: \underline{\underline{\varepsilon}}^{p}=\boldsymbol{p} \dot{\gamma}(\boldsymbol{\eta}-\boldsymbol{\psi})$. Since, $\boldsymbol{p} \dot{\gamma}>\mathbf{0}$, for $\boldsymbol{\varpi}$ to be positive, it is necessary and sufficient that $\boldsymbol{\eta}-\boldsymbol{\psi}(\boldsymbol{\eta}, \boldsymbol{\alpha})$ is positive for all $\boldsymbol{\eta}>\mathbf{0}$ and $\boldsymbol{\alpha}$.

This condition is verified for all the studied models in this paper.
In sum, a soil constitutive model in this presented family is fully defined with Equation (9) and the two functions $\boldsymbol{\phi}(\boldsymbol{p}, \boldsymbol{\alpha})$ and $\boldsymbol{\psi}(\boldsymbol{\eta}, \boldsymbol{\alpha})$. The yield criterion is $\max \left[-\boldsymbol{p}, \boldsymbol{p}-\boldsymbol{p}_{\boldsymbol{c}}(\zeta), \boldsymbol{q}-\boldsymbol{\phi}(\boldsymbol{p}, \boldsymbol{\alpha})\right.$.

In the following, three critical state models will be studied and compared. At this point, we would like to underline that "a critical state stands for a stress state where shear strains develop without any change of volume and effective stresses" (Schofield \& Wroth, 1968). Such a critical state is reached at a stress ratio $\eta=M$, where $M$ is the slope of the critical state line in the stress space ( $p-$ $q$ ). However, in practice, during a classic triaxial test, the critical state is reached in an "asymptotic" way (when the shear strain is large enough). Hence, the plastic criterion $\boldsymbol{f}(\underline{\underline{\boldsymbol{\sigma}}}, \boldsymbol{\alpha})$ must verify the condition $\lim \partial_{\gamma} f=0$ when $\lim \gamma=\infty$.

\subsection{Modified Cam Clay model}

The MCC model, the prototype of critical state soil models, is taken as a reference in this comparison. Its elliptical yield function, given in Equation (14), evolves with the preconsolidation pressure (volumetric hardening mechanism).

$$
\boldsymbol{F}\left(\underline{\underline{\sigma}}, p_{c}\right)=\left(\frac{\boldsymbol{q}}{M}\right)^{2}+\boldsymbol{p}\left(\boldsymbol{p}-\boldsymbol{p}_{c}\right)
$$

The MCC model flow rule written in the incremental form is given by Equation (15).

$$
\frac{\dot{\zeta}}{\dot{\gamma}}=\frac{\eta^{2}-M^{2}}{2 \eta}
$$

\subsection{Clay and Sand Model}

To overcome the failure of critical state based soil models to simulate the softening and dilatancy of dense sands, $\mathrm{Yu}$ (1998) proposed the unified clay and sand model (CASM). It is presented in this work in terms of preconsolidation pressure. Its yield function is given in Equation (16).

$$
F\left(\underline{\underline{\sigma}}, p_{c}\right)=\left(\frac{q}{M p}\right)^{n}+\frac{1}{\ln (r)} \ln \left(\frac{p}{p_{c}}\right)
$$

where $\boldsymbol{n}$ and $\boldsymbol{r}$ are model parameters.

The original CASM version published by $\mathrm{Yu}$ (1998) used a Rowe stress-dilatancy rule, written in Equation 
(17), that was not particularly realistic for stress paths with low stress ratios.

$$
\frac{\dot{\zeta}}{\dot{\gamma}}=\frac{9(M-\eta)}{9+3 M-2 M \eta}
$$

Later, $\mathrm{Yu}$ (2006) proposed the flow rule considered in this study and given by Equation (18).

$$
\frac{\dot{\zeta}}{\dot{\gamma}}=\frac{\eta^{n}-M^{n}}{m \eta^{n-1}}
$$

where $m$ is a model parameter.

In comparison to the MCC model, with a nonassociative flow rule and three additional parameters, CASM provides a better simulation of the behavior of overconsolidated clay. These two models only use volumetric plastic strain hardening. However, integrating both volumetric and deviatoric plastic strain hardening may be more suitable for the simulation of shear stress paths or mainly deviatoric loadings. Furthermore, the nonlinearity of the stress-strain soil response before failure is not captured by either model. To overcome this drawback, the proposed model integrates two hardening mechanisms and an early start of plasticity with only one yield surface.

\subsection{Proposed model (SCSM)}

The shear critical state model (SCSM) reduces the shear resistance overestimated by the MCC model for stress paths with low mean pressure and uses deviatoric hardening in the framework of critical state theory. Its yield surface has an elliptical shape, defined by Equation (19), that evolves with the volumetric and deviatoric hardening mechanisms.

$$
F\left(\underline{\underline{\sigma}}, p_{c}\right)=\left(\frac{q}{M_{\gamma} p}\right)^{2}+\ln \left(\frac{p}{p_{c}}\right)
$$

The volumetric hardening is given by the evolution of the preconsolidation pressure with the plastic volumetric strain according to Equation (9) and the introduced shear hardening is controlled by $M y$ given by Equation (20):

$$
M_{\gamma}=\frac{M_{\infty} \gamma+M_{0} a}{\gamma+a}
$$

where $M_{\infty}, \boldsymbol{M}_{\mathbf{0}}$ and $a$ are model parameters. The difference between $\boldsymbol{M}_{\mathbf{0}}$ and $\boldsymbol{M}_{\infty}$ ensures a smooth transition to plastic failure in critical state. The function $M y$ has a finite

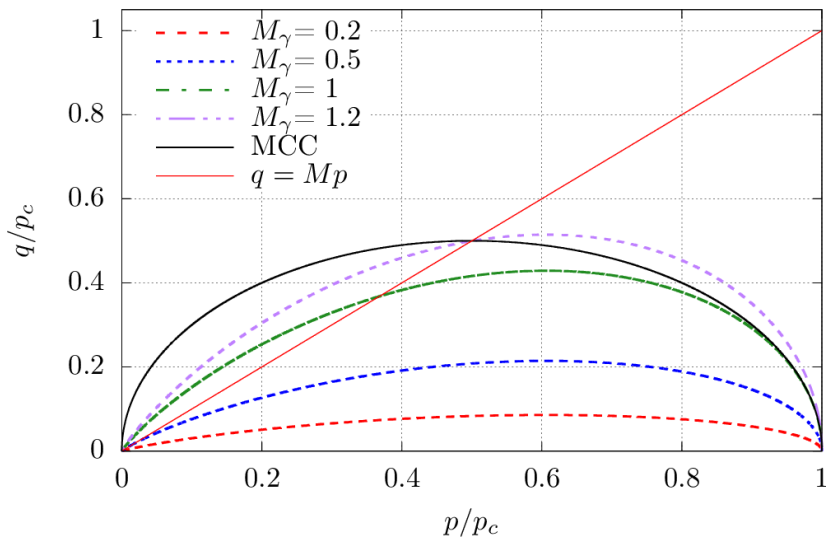

Figure 2: Shear yield surface hardening with plastic shear strains.

limit $M_{\infty}$ when $y$ tends towards infinity that ensures the condition of critical state as defined in the end of Section 2.3.

Figure 2 shows the evolution of the yield surface with the deviatoric hardening function $M y$. For comparison, the yield surface of the MCC model, with $M=1$, is drawn in a solid line on the same figure.

The evolution of the plastic strains is determined by the flow rule written as a function of the stress ratio $\boldsymbol{\eta}=\boldsymbol{q} / \boldsymbol{p}$ as follows :

$$
\frac{\dot{\zeta}}{\dot{\gamma}}=\frac{\eta^{l}-M^{l}}{l \eta^{l-1}}
$$

where $l$ is a model parameter. The choice of this flow rule ensures zero volumetric plastic strains for $\eta=\mathrm{M}$.

\subsubsection{SCSM features}

The proposed model was implemented in the in-house finite element code VIPLEF (Tijani, 1996). Its performance will be studied in the following. All the presented simulations were conducted under a uniform stress state. A sensitivity study was performed through the simulation of fictive (theo-retical) triaxial tests to assess the model features and to emphasize the wide range of the model's response with different parameters. The reference soil parameters are summarized in Table 1. The first four Camclay parameters are taken similar to those of London clay based on the work of Gasparre (2005).

The reference test is characterized by an initial preconsolidation pressure $p_{\mathrm{c}}$ of $900 \mathrm{kPa}$ and an initial mean pressure of $300 \mathrm{kPa}$. The corresponding

In all the figures representing triaxial tests in what follows, $\varepsilon_{\mathrm{a}}$ stands for axial strain and $\varepsilon_{\mathrm{v}}$ is the volumetric 


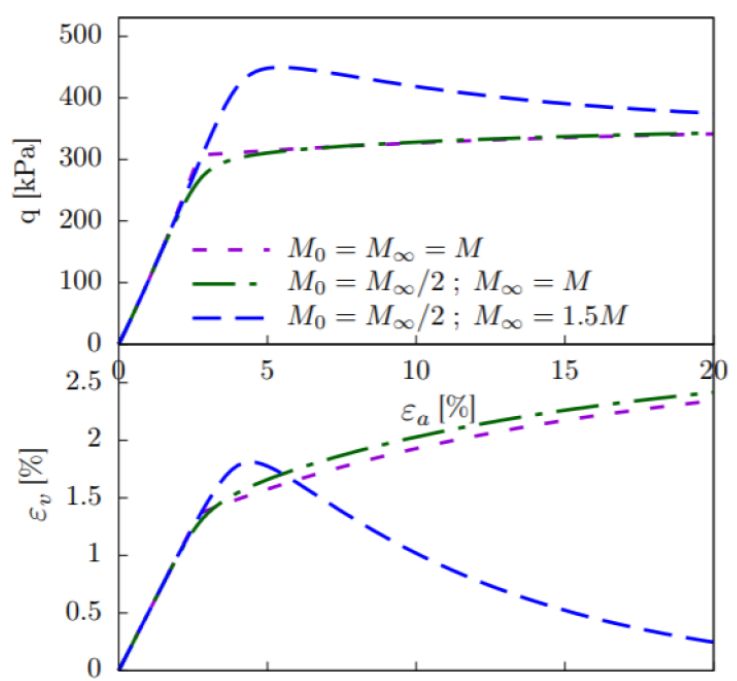

(a) Variation of the model's response with parameters $M_{0}$ and $M_{\infty}$

Figure 3: Response of a drained triaxial test.

Table 1: Reference material parameters.

\begin{tabular}{cccccccc}
\hline$v$ & $\kappa$ & $\lambda$ & $M$ & $M_{0}$ & $M_{\infty}$ & $a$ & $I$ \\
\hline 0.25 & 0.08 & 0.168 & 0.85 & 0.17 & 1.27 & 0.005 & 2 \\
\hline
\end{tabular}

strain with the sign convention that $\varepsilon_{\mathrm{v}}$ is positive for contraction.

The parameter $M_{0}$ indicates the start of yielding and the parameter $M_{\infty}$ defines the soil resistance, whereas $M$ is the slope of the critical state line. Thus, a ratio between $M_{\infty}$ and $M$ greater than one induces a softening soil response as shown in Figure 3a. The difference between $M_{0}$ and $M_{\infty}$ does not greatly modify the deformation response but results in a smoother stress-strain response.

The deviatoric hardening constant $a$ changes in a range between 0.05 and 0.0001 . A small value of the parameter $a$ produces more irreversible strains before failure as illustrated in Figure $3 \mathrm{~b}$.

Figure 4a shows that a change in the flow rule parameter $l$ does not in-fluence the stress-strain response and only helps to adjust the deformation response. In the MCC model this parameter is equal to 2 for all types of soil.

The change in the SCSM response with initial stress conditions, or the $O C R$ values given in Figure $4 \mathrm{~b}$, is in good agreement with experimental observations.

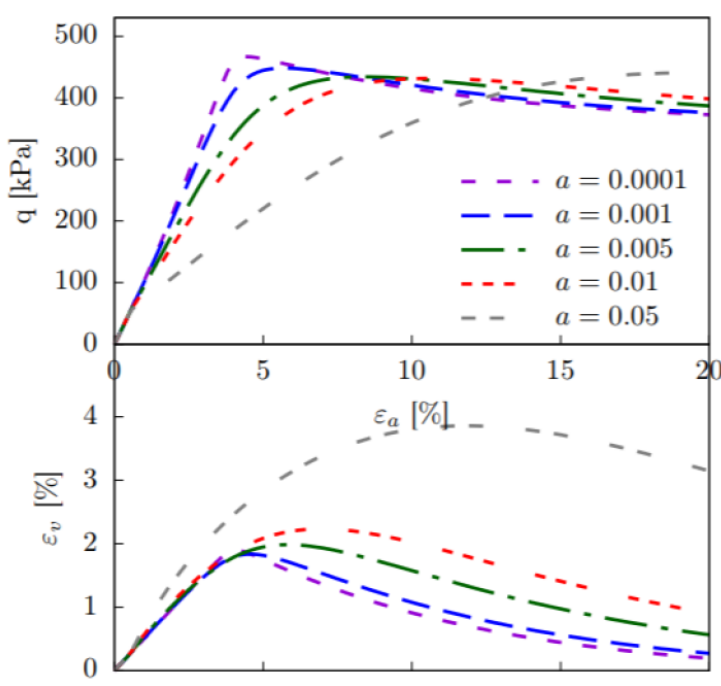

(b) Variation of the model's response with parameter $a$

\subsection{Overview of studied models}

The MCC model, CASM and the proposed SCSM are presented in Table 2 with the notations of the standardized formulation described in Section 2.3 and are then compared afterwards. The parameters $p_{\mathrm{co}}, \lambda, \kappa$ and $v$ in addition to the slope of the critical state line $M$ are common to all the models in this family.

The functions $\phi$ and $\psi$ defining each soil model are given in Table 2. Only the additional parameters to $p_{c 0}$, $\lambda, \kappa$ and $v$ are written in this table. Furthermore, the last line of the table features the range of the choice of some parameters to verify that the intrinsic dissipation is positive. This condition was reduced to $\eta-\boldsymbol{\psi}(\boldsymbol{\eta}, \boldsymbol{\alpha})>\mathbf{0}$, as explained in Section 2.3.

The models' parameters can be defined according to the methodology described in the subsequent section.

\subsubsection{Determination of the models' parameters}

The proposed model (SCSM) has 9 parameters $: v, p_{\mathrm{c} 0}, \kappa$, $\lambda, M, M_{0}, M_{\infty}, a$ and $l$. The common parameters to MCC, $\kappa$, $\lambda$ and $p_{\text {c0 }}$ can be determined by an isotropic compression test. $\kappa$ is the slope of the swelling line of the isotropic compression curve in $e-\ln p$ plane and $\lambda$ is the slope of the normal consolidation line in the same plane. The slope of the critical state line, $M$, is classically determined with at least two compression triaxial tests. $M_{0}$ and $M_{\infty}$ can also be determined with a classical compression triaxial test 


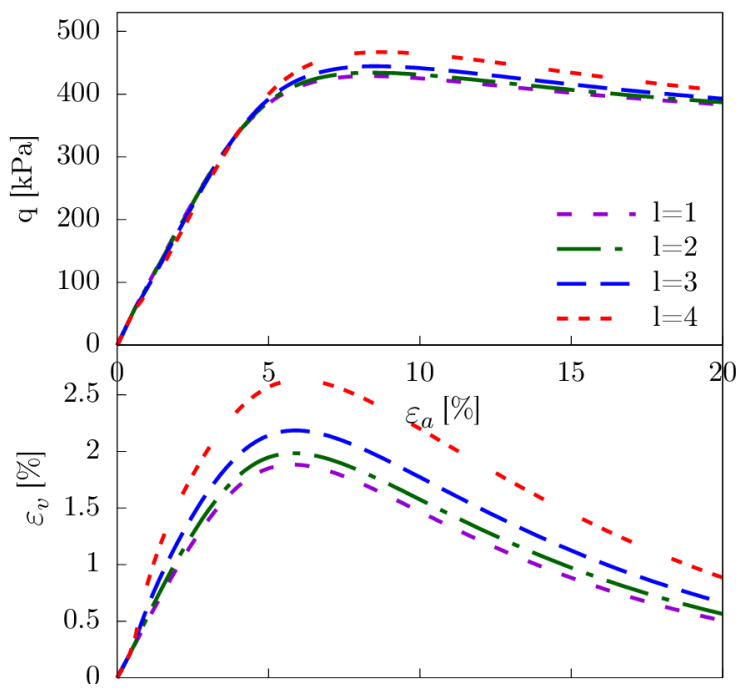

(a) Variation of the model's response with parameter I

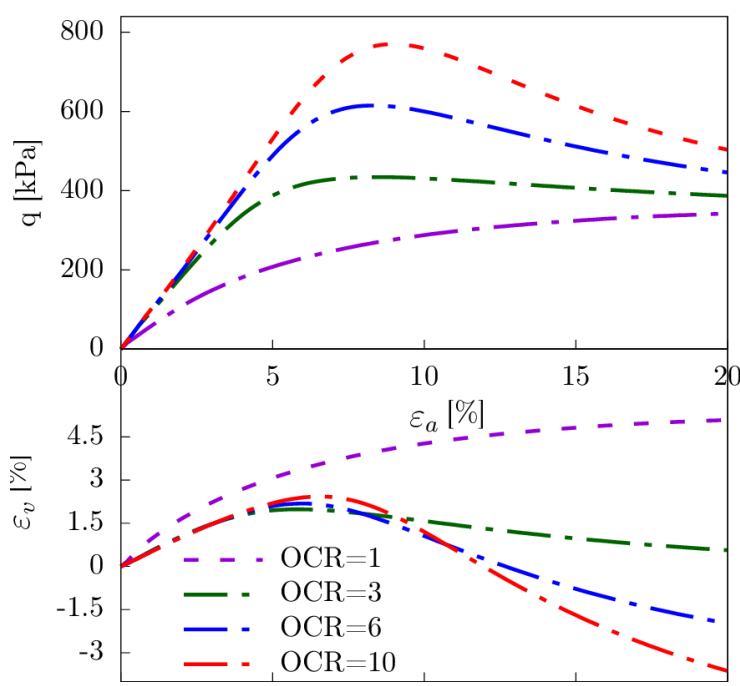

(b) Variation of the model's response with OCR

Figure 4: Response of a drained triaxial test.

Table 2: Studied models standardized formulation.

\begin{tabular}{llll}
\hline & MCC & CASM & SCSM \\
\hline$\alpha(p, \alpha)$ & $\zeta$ & $\zeta$ & $(\zeta, \gamma)$ \\
& $M p\left[\left(\frac{p_{c}}{p}-1\right)\right]^{1 / 2}$ & $M p\left[\frac{1}{\ln r} \ln \left(\frac{p_{c}}{p}\right)\right]^{1 / n}$ & $M_{\gamma}(\gamma) p\left[\ln \left(\frac{p_{c}}{p}\right)\right]^{\frac{1}{2}} ;$ \\
$\psi(\eta, \alpha)$ & $\frac{\eta\left[1-\left(\frac{M}{\eta}\right)^{2}\right]}{2}$ & $\frac{\eta\left[1-\left(\frac{M}{\eta}\right)^{n}\right]}{m}$ & $M_{\gamma}(\gamma)=\frac{M_{\infty} \gamma+M_{0} a}{\gamma+a}$ \\
Parameters & $M$ & $M, r, n, m$ & $\eta\left[1-\left(\frac{M}{\eta}\right)^{l}\right]$ \\
$\eta-\psi(\eta, \alpha)>0$ & & $m>1$ & $l$
\end{tabular}

but the easiest way is based on a triaxial shear stress at constant mean pressure. In such a test, the volumetric strain is zero before the plasticity. This transition is given by $M_{0}$, and the maximum shear stress gives $M_{\infty}$. The parameter $a$ controls the angle of the nonlinear stressstrain response in that test. The flow rule is controlled by the parameter $l$ which is deter-mined from the curve of the volumetric strain versus the axial one for any triaxial test.

The appropriate approach to determine the model parameters based on laboratory tests is described below.

To identify the model parameters, for each soil, the minimum data are the results of two laboratory tests :

1. an isotropic test where the effective mean pressure $p$ and the volumetric strain $\varepsilon_{\mathrm{v}}$ are measured,

2. a triaxial test where $p, q, \varepsilon_{\mathrm{v}}$ and the axial strain $\varepsilon_{\mathrm{a}}$ are measured.

Both tests should be carried out as far as possible with measurements recorded at a relatively high frequency. The setting of parameters values can generally be established in two steps. In the first step, the stress and strain states are considered uniform in the test sample. The friction between the two boundaries of the specimen and the porous stones (drained test) or the pedestal and the top cap (undrained test) is neglected. In the second step, the side effects are taken into account and a finite element simulation of the specimen is required. The estimation of the parameters values can then be refined by linking a finite element code to an adjustment code. The fitted 
parameters of the first step are used as a starting point of the refinement. Only the first step is considered in the following for the three soil models presented in Table 2.

The isotropic test is used to fit the parameters $\kappa^{*}, \lambda^{*}$ and $p_{\mathrm{c} 0}$. The values are fitted such as the measurements verify, in the least square sense, the following equations (deducted from equations (7) and (8) ) :

$$
\varepsilon_{v}=\boldsymbol{\kappa}^{*} \ln \left(\frac{p}{p_{0}}\right)+\left(\lambda^{*}-\boldsymbol{\kappa}^{*}\right)\left\langle\ln \left(\frac{p}{p_{c 0}}\right)\right\rangle
$$

where the Macaulay brackets $\langle$.$\rangle are defined for any real$ number $\boldsymbol{x}$ as $\langle\boldsymbol{x}\rangle=(\boldsymbol{x}+|\boldsymbol{x}|) / \mathbf{2}$.

The triaxial tests are generally performed at constant confining pressure (case 1: $\delta p=1 / 3 \delta q$ ) or at constant mean pressure (case 2: $\delta p=0$ ).

According to Equation (10), in both cases, the volumetric elastic strainIs

$$
\varepsilon_{v}^{e}=\kappa^{*} \ln \left(\frac{p}{p_{0}}\right)
$$

The axial elastic strain is:

$$
\left\{\begin{array}{l}
\operatorname{case} 1: \varepsilon_{a}^{e}=\frac{\kappa^{*}}{1+2 v} \ln \left(\frac{p}{p_{0}}\right) \\
\text { case } 2: \varepsilon_{a}^{e}=\frac{2(1+v) \kappa^{*}}{9(1-2 v)} \frac{q}{p_{0}}
\end{array}\right.
$$

Equation (24) is first used to fit the value of the Poisson's ratio $v$ on the first part of the triaxial test results. They are then employed to calculate the plastic strains by subtracting the elastic strains from the total measured strains $\varepsilon_{\mathrm{a}}$ and $\varepsilon_{\mathrm{v}^{*}}$. The hardening variables $\zeta$ and $\gamma$ are then calculated from the plastic strains. We end up with a file containing $(p, q, \zeta, \gamma)$ that is processed so that $\gamma$ is zero for the first part (elastic part) and then strictly increasing in the second part. We verify that $\gamma=0$ when $\zeta=0$ and only use the part where $y>0$. The last part is completed by calculating the stress ratio $\eta$ and $\delta \zeta / \delta y$. The remaining parameters of $\phi$ and $\psi$ are then fitted such as : $\frac{\delta \zeta}{\delta \gamma}=\psi(\eta, \alpha)$ and $q=\phi(p, \alpha)$

\section{Comparison of the model responses}

The proposed model responses under triaxial compression conditions are first validated against published laboratory results on London clay, Weald clay and Boom clay. The performance of the model to simulate undrained and drained soil responses at different overconsolidation ratios is assessed in comparison to the MCC model and CASM on triaxial tests. Since all three models are critical state models and have similar elastic and volumetric hardening characteristics, the focus is on the improvement provided by the deviatoric hardening mechanism.

All the samples were isotropically consolidated and then unloaded up to an initial mean pressure $p_{0}$. The consolidation state of each soil sample is defined by the overconsolidation ratio $(O C R)$ which is the ratio of the initial preconsolidation pressure $p_{\mathrm{co}}$ to the initial mean pressure $p_{0}\left(O C R=p_{c 0} / p_{0}\right)$.

The threeconsidered clays parameters are summarized in Table 3 con-taining the common MCC parameters and SCSM parameters $\left(M_{0}, M_{\infty}, a\right.$ and $\left.l\right)$. The missing CASM parameters are summarized in Table 4. It should be noted that MCC model parameters are taken from the respec-tive work references (Gasparre, 2005; Bishop \& Henkel, 1957; Sultan, Cui, \& Delage, 2010) as mentioned by the original authors for each clay. The SCSM and CASM parameters were adjusted to obtain the best possible fit.

\subsection{Undrained triaxial tests}

The model undrained response was validated on data from undrained triax-ial tests performed by Gasparre (2005) in her work on reconstituted London clay. For these simulations, London clay reconstituted samples were sheared at overconsolidation ratios of $O C R=1,3$ and 12 , and the corresponding initial mean pressures are respectively $p_{0}=485,200$ and $50 \mathrm{kPa}$.

The simulation results of these three undrained triaxial tests with the three studied models are illustrated in Figure 5. The undrained condition is modeled by imposing a zero volumetric strain, i.e., $. \operatorname{tr}(\underline{\dot{\varepsilon}})=\mathbf{0}$ The values of deviatoric stress at failure are satisfactorily predicted with the proposed model for different overconsolidation ratios as shown. In contrast, the MCC model and CASM predict a much higher shear resistance for the heavily overconsolidated sample with an overconsolidation ratio of 12. Both models give similar good agreement with experimental results for the normally consolidated sample whereas for the triaxial test with $O C R=3$, SCSM shows a better agreement with a smooth transition from elasticity to plasticity as the good reproduction of the non linear stress-strain response for the heavily overconsolidated sample with $O C R=12$. 


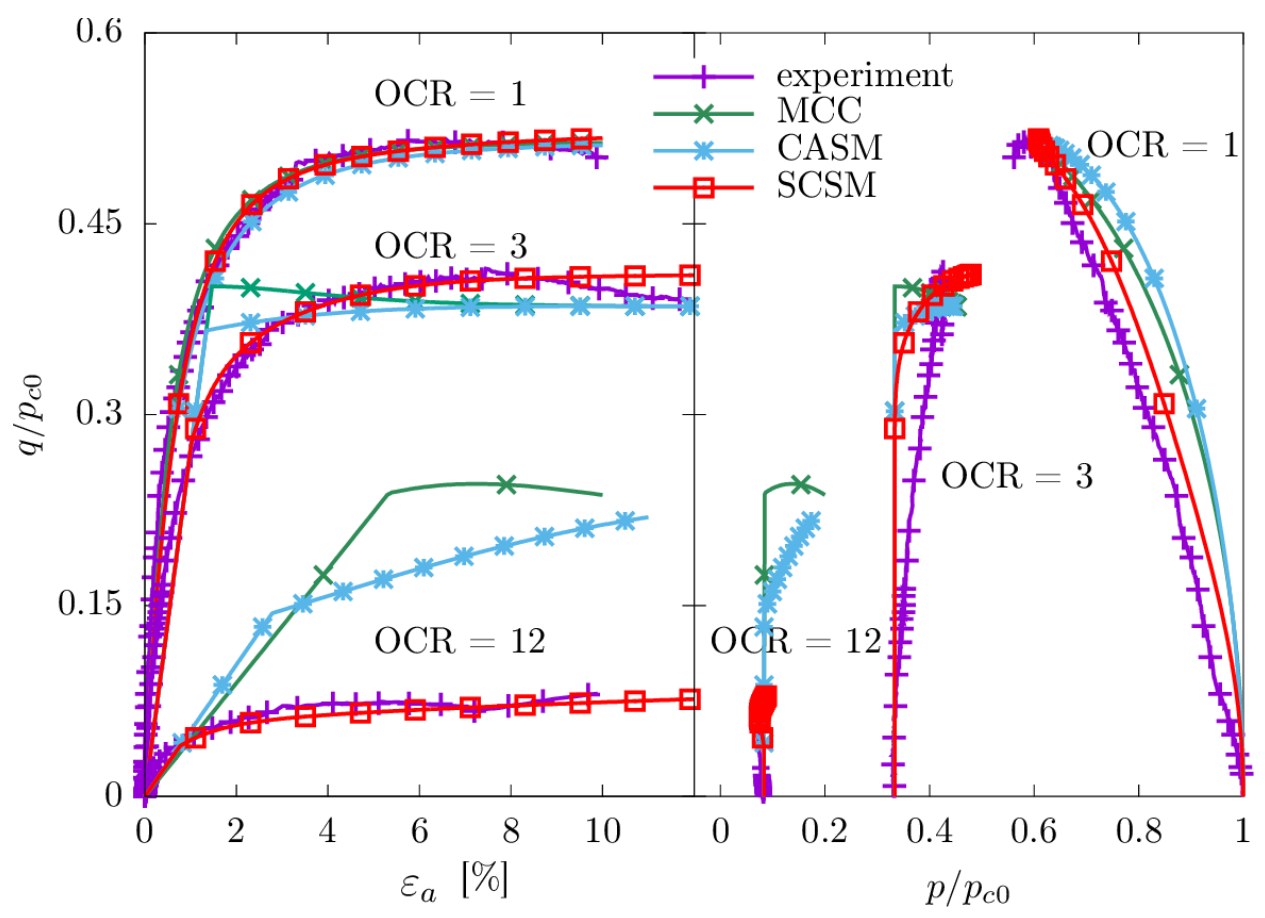

Figure 5: Simulation of undrained compression of London clay for normally consolidated and overconsolidated states.

Table 3: MCC and SCSM parameters for different clays.

\begin{tabular}{lllllllll}
\hline Model parameter & $\boldsymbol{V}$ & $\boldsymbol{K}$ & $\boldsymbol{\lambda}$ & $\boldsymbol{M}$ & $\boldsymbol{M}_{\mathbf{0}}$ & $\boldsymbol{M}_{\infty}$ & $\boldsymbol{a}$ & $\boldsymbol{l}$ \\
\hline London clay & 0.25 & 0.064 & 0.168 & 0.85 & 0.8 & 1.1 & 0.005 & 2 \\
Weald clay & 0.2 & 0.025 & 0.093 & 0.9 & 0.7 & 1.1 & 0.001 & 2 \\
Boom clay & 0.3 & 0.017 & 0.03 & 0.71 & 0.4 & 0.8 & 0.0025 & 2 \\
\hline
\end{tabular}

Table 4: CASM parameters for different clays.

\begin{tabular}{llll}
\hline Model parameter & $r$ & $n$ & $m$ \\
\hline London clay & 2.0 & 1.8 & 2.5 \\
Weald clay & 2.714 & 4.5 & 2.9 \\
Boom clay & 2.4 & 2.0 & 2.0 \\
\hline
\end{tabular}

\subsection{Drained triaxial tests}

\subsubsection{Weald clay}

Test data from the classic series of tests on Weald clay realized in Imperial College London by Bishop and Henkel (1957) has been used by Yu (1998) for the validation of the CASM. The simulation of two drained triaxial compression tests is presented to assess the performance of SCSM. The first test is performed on a normally consolidated sample with $O C R=1$ and the second on a heavily overconsolidated sample with $O C R=24$.

The initial state of the normally consolidated sample is characterized by an initial mean pressure $p_{0}$ of $207 \mathrm{kP}$ $a$ and an initial void ratio $e_{0}$ of 0.632 , whereas for the overconsolidated sample, $p_{0}=34.5 \mathrm{kP} a$ and $e_{0}=0.617$.

The model parameters used for the simulation are summarized in Tables 3 and 4.

The MCC model gives a satisfactory simulation of the behavior of the normally consolidated Weald clay just as CASM and SCSM as shown in the Figure 6a. However, Figure $6 \mathrm{~b}$ shows that the MCC model excessively overestimates the shear resistance and the plastic strains of the overconsol-idated sample. CASM and SCSM succeed in the prediction of the shear resistance of Weald clay for both $O C R=1$ and $O C R=24$. The SCSM results in a smooth transition from elasticity to plasticity with a slightly better fit for the volumetric strains in comparison to the CASM. 


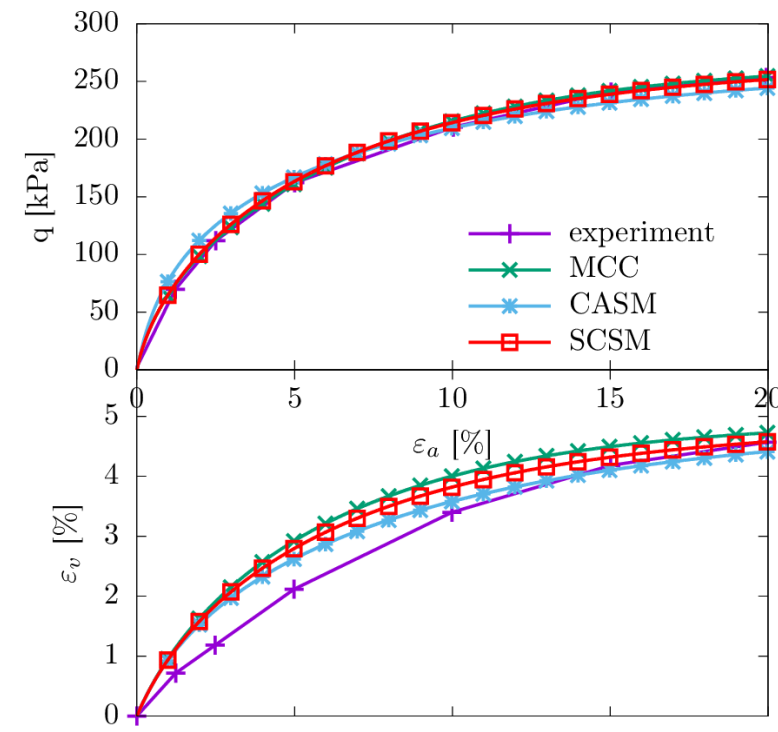

(a) $\mathrm{OCR}=1$

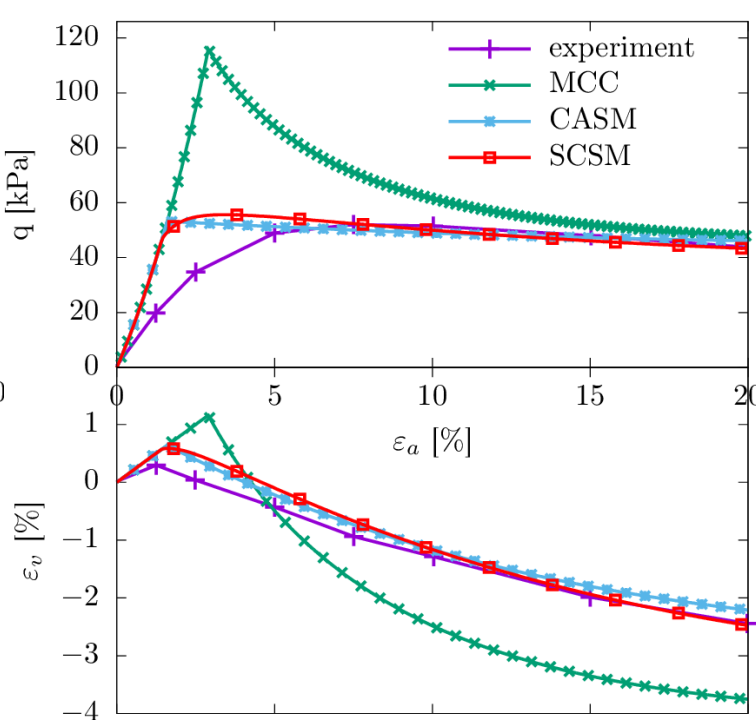

(b) $\mathrm{OCR}=24$

Figure 6: Simulation of drained compression of Weald clay for normally consolidated and overconsolidated states.

Hence, the improvement with respect to the CASM seems negligible for these Weald clay tests.

\subsubsection{Boom clay}

An underground research laboratory devoted to research into nuclear waste disposal was excavated in a deposit of Boom clay. This overconsolidated clay has been the subject of many studies. Sultan et al. (2010) conducted several triaxial tests on Boom clay for different overconsolidation ratios. The Boom clay parameters, summarized in Tables 3 and 4, were defined based on the extensive study of this material in the thesis of DAO (2015). The initial mean pressure of the first slightly overconsolidated test with $O C R=1.8$ is $5 \mathrm{MPa}$ whereas it is $0.9 \mathrm{MPa}$ for the overconsolidated test with $O C R=10$.

The simulation of the lightly overconsolidated Boom clay sample with the three studied constitutive models is given in Figure 7a. The SCSM pre-dicts a smooth transition from elasticity to plasticity in agreement with the experimental data, contrary to the CASM and MCC models, which predict a sharp transition. The earlier yielding with the CASM in comparison to the MCC model results in an underestimation of the maximal shear stress. For the simulation of the response of the the overconsolidated sample, the MCC overestimates the shear resistance and the dilatancy as illustrated in Figure 7b. The SCSM gives the best prediction of the nonlinearity of the stress strain response for both overconsolidation ratios without excessively overestimating the volumetric strains.

The disparities between the responses of the three considered soil models are emphasized with the simulation of a fictive (theoretical) pure shear triaxial test at constant effective pressure where the deviatoric hardening mechanism of SCSM is activated for different overconsolidation ratios. The same Boom clay parameters summarized in Table 4 were used to simulate a fictive shear triaxial test at two constant effective pressures $p$ $=5 \mathrm{MPa}$ and $p=0.9 \mathrm{MPa}$ representing a pure shear at two overconsolidation ratios $O C R=1.8$ and $O C R=10$, respectively. The simulation of a stress path controlled triaxial test is not straightforward. In fact, a displacement load resulting in the required stress path had to be imposed to avoid any aberration as detailed in Section A. The responses of the three soil models to these two simulations are given in Figures $8 \mathrm{a}$ and $8 \mathrm{~b}$.

In this test, at constant mean pressure, all the volumetric strains are plastic strains. Plasticity occurs earlier with the SCSM in comparison to the MCC model and CASM. For $p>p c / 2$, the response is contractional with the three soil models as shown in Figure 8a while it is dilatant for $p<p c / 2$ as illustrated in Figure $8 \mathrm{~b}$. In the simulation of the classic compression triaxial test with $O C R=1.8$ shown in Figure $7 \mathrm{a}$, all the models have ap-proximatively the same strain response while the discrepancy is greater on the test with $p=5 \mathrm{MPa}$. The volumetric strain predicted with the SCSM is indeed almost twice as large as with the 


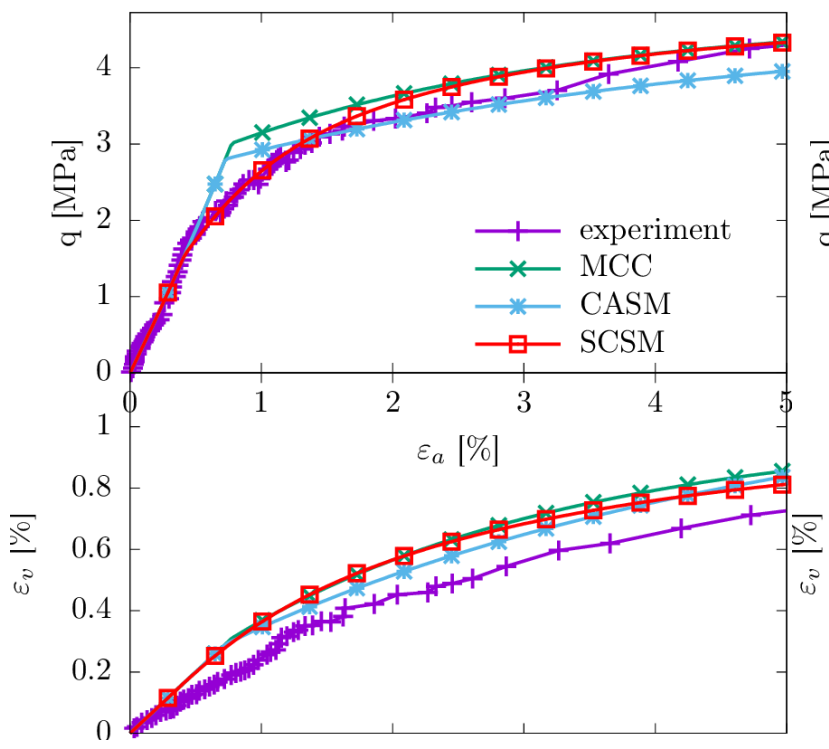

(a) OCR = 1.8

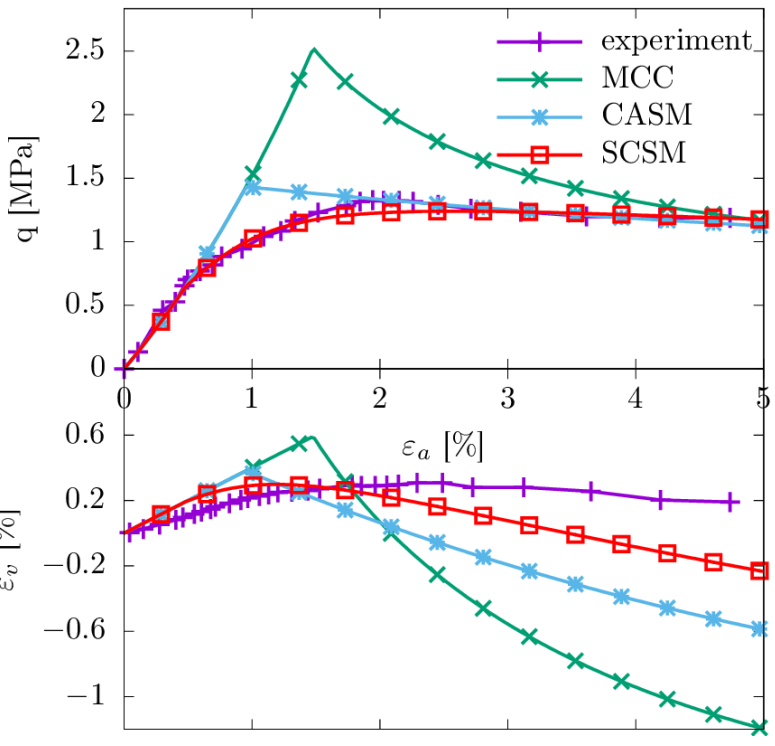

(b) $\mathrm{OCR}=10$

Figure 7: Simulation of drained compression of Boom clay for normally consolidated and overconsolidated states.

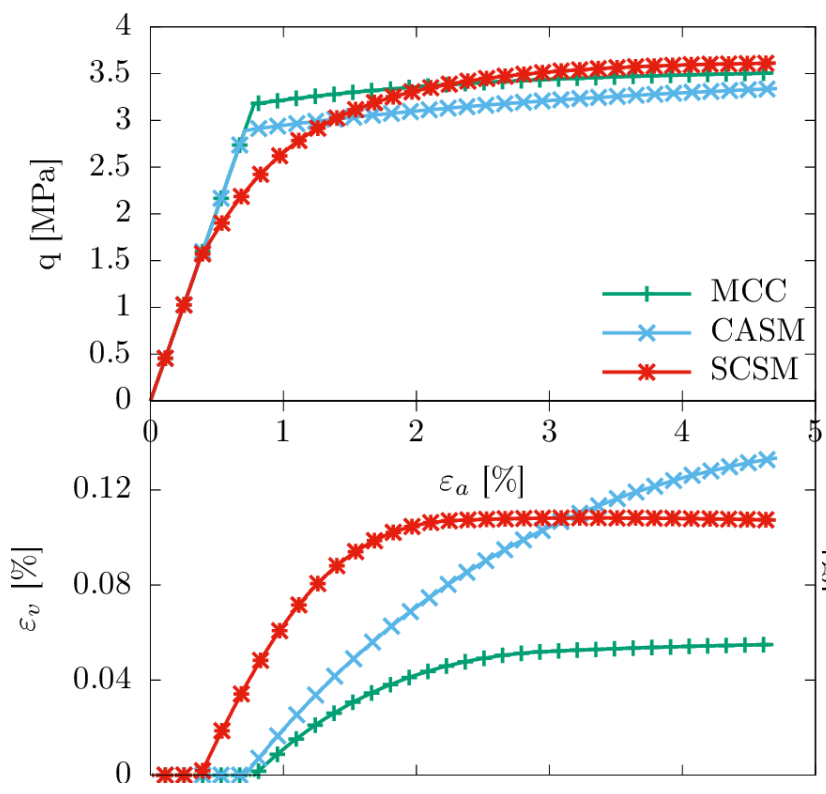

(a) $p=5 M P$ a

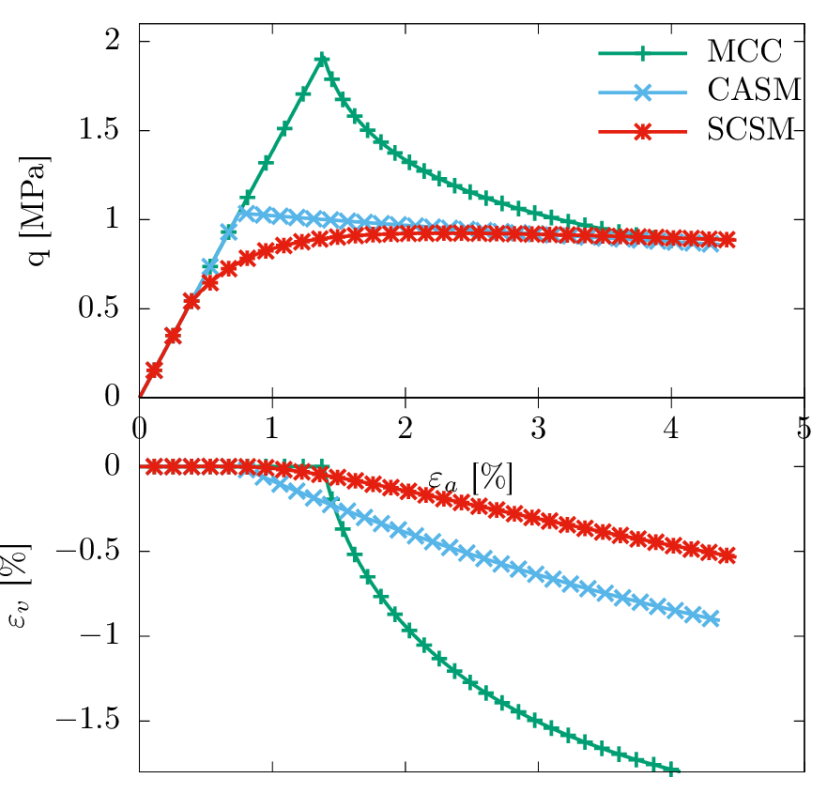

(b) $p=0.9 \mathrm{MPa}$

Figure 8: Simulation of pure triaxial shearing at constant effective pressure $p$.

MCC model. The CASM pre-dicts higher volumetric strains for this test.

Parameter calibration for a classic triaxial test where the three soil mod-els have approximatively the same response for an initial stress state with $p>p c / 2$ results in different predictions for a pure shear test at the same initial effective pressure. This shows that the calibration of a soil constitu-tive model parameters based on a classic triaxial test does not necessarily lead to similar responses for different stress paths. In practice, soil elements around a geotechnical structure are subjected to stress paths other than those followed in a classic triaxial test. The reproduction of specific stress paths in the laboratory is then required. Particularly, purely deviatoric stress paths 
are of interest for the development of soil constitutive models because they permit to measure the volumetric strain created by the sole deviatoric stress (Hattab \& Hicher, 2004) and then have a better under-standing of soil dilatancy.

For example, stress paths around an excavation have been the subject of several studies. Based on centrifuge model tests and finite element analysis, Mair (1979) showed that tunneling induced stress paths are mainly deviatoric. Barla (1999) proposed and performed laboratory tests, mainly deviatoric, reproducing expected stresses at different points of an excavation for an isotropic intial stress state. In this extreme case, the loading is purely deviatoric around a circular excavation (Panet, 1995; Gilleron \& Bourgeois, 2016).

\subsection{Shear triaxial tests at constant $p$}

To explore the soil response under purely deviatoric loading, triaxial tests were performed respecting the loading scheme described in Figure 9 on a gray marl. The first part reproduces a pure deviatoric stress followed by an unloading part since it may be induced in the soil under some circumstances in geotechnical works. Isotropic compression is performed to verify the impact of shear hardening on volumetric hardening. This Piacenzian marl was core drilled in the south of France. Its yield surface has been studied and characterized by Serratrice (2002). The use of samples of this marl helped in the definition of the loading paths of the realized triaxial tests where the shear stress limit was chosen so that the specimen yields before unloading.

The proposed model has been used to predict the behavior of this over-consolidated marl. Three tests were conducted where the shearing was realized at different constant mean pressures, of 490, 1000 and $1800 \mathrm{kPa}$. The unloading of the pure shear was followed by isotropic compression until $4000 \mathrm{kPa}$.

The simulations of these tests were performed by applying a controlled displacement that results in the required stress path. The loading-unloading criterion is given by the sign of the mechanical power defined as the scalar product of the stress tensor and the strain rate tensor. A positive power defines a loading path where energy is supplied to the soil. In contrast, an unloading path is defined by a negative power. These gray marl parameters are summarized in Table 5 for all three studied models.

The first test (Test1) simulation in Figure 10a shows that the MCC model and the CASM overestimate the shear

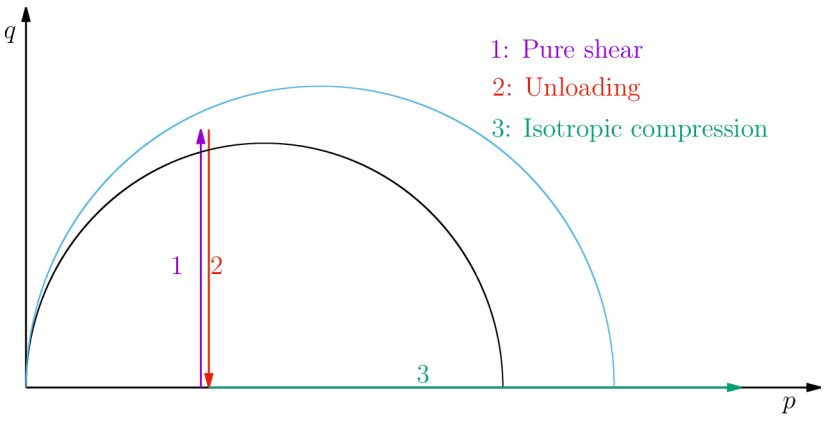

Figure 9: Loading path..

Table 5: MCC, SCSM and CASM parameters for the grey marl.

\begin{tabular}{llllllllllll}
\hline & \multicolumn{1}{c}{ MCC } & \multicolumn{4}{c}{ SCSM } & \multicolumn{3}{c}{ CASM } \\
\hline$v$ & $\boldsymbol{K}$ & $\lambda$ & $\boldsymbol{M}$ & $\boldsymbol{M}_{\mathbf{0}} \boldsymbol{M}_{\infty}$ & $\boldsymbol{a}$ & $\boldsymbol{l}$ & $\boldsymbol{r}$ & $\boldsymbol{n}$ & $\boldsymbol{m}$ \\
\hline 0.3 & 0.017 & 0.04 & 0.98 & 0.4 & 1.3 & 0.0017 & 2 & 1.8 & 3.5 & 3.0 \\
\hline
\end{tabular}

resistance at small mean pressures or by the dry side of the critical state line. The simulation of this test with the proposed model generates plastic strains in contrast to the MCC model and the CASM which predict that the shearing remains in the elastic region. MCC and CASM forecast the maximum shear response of Test 2 with a sharp transition from elasticity to plasticity as shown in Figure 10b. In contrast, the SCSM reproduces the nonlinearity of the shear loading with a smooth transition to plasticity. The three models reproduce the stress-strain response of the normally consolidated sample satisfactorily as illustrated in Figure 10c.

The volumetric strains corresponding to the drained water volume are generated for the three tests at the beginning of the shearing whereas the soil constitutive models simulate an elastic part where the volumetric strains are zero. Theoretically, all volumetric strains at constant mean pressure are plastic strains.

The unloading response is not correctly simulated by any of the chosen soil models. To investigate the cause of the non linearity of the unloading, the influence of the stress unloading rate was studied with a hydromechanical test simulation. The increase in the pore pressure with a very low permeability (approximately $10^{-9} \mathrm{~m} / \mathrm{s}$ ) was negligible and had an insignificant influence on the unloading simulated response. Hence, it has been verified that these tests were conducted at a sufficiently low rate to guarantee drained conditions.

Since hydromechanical coupling could not enhance the simulation of the unloading response, the influences 

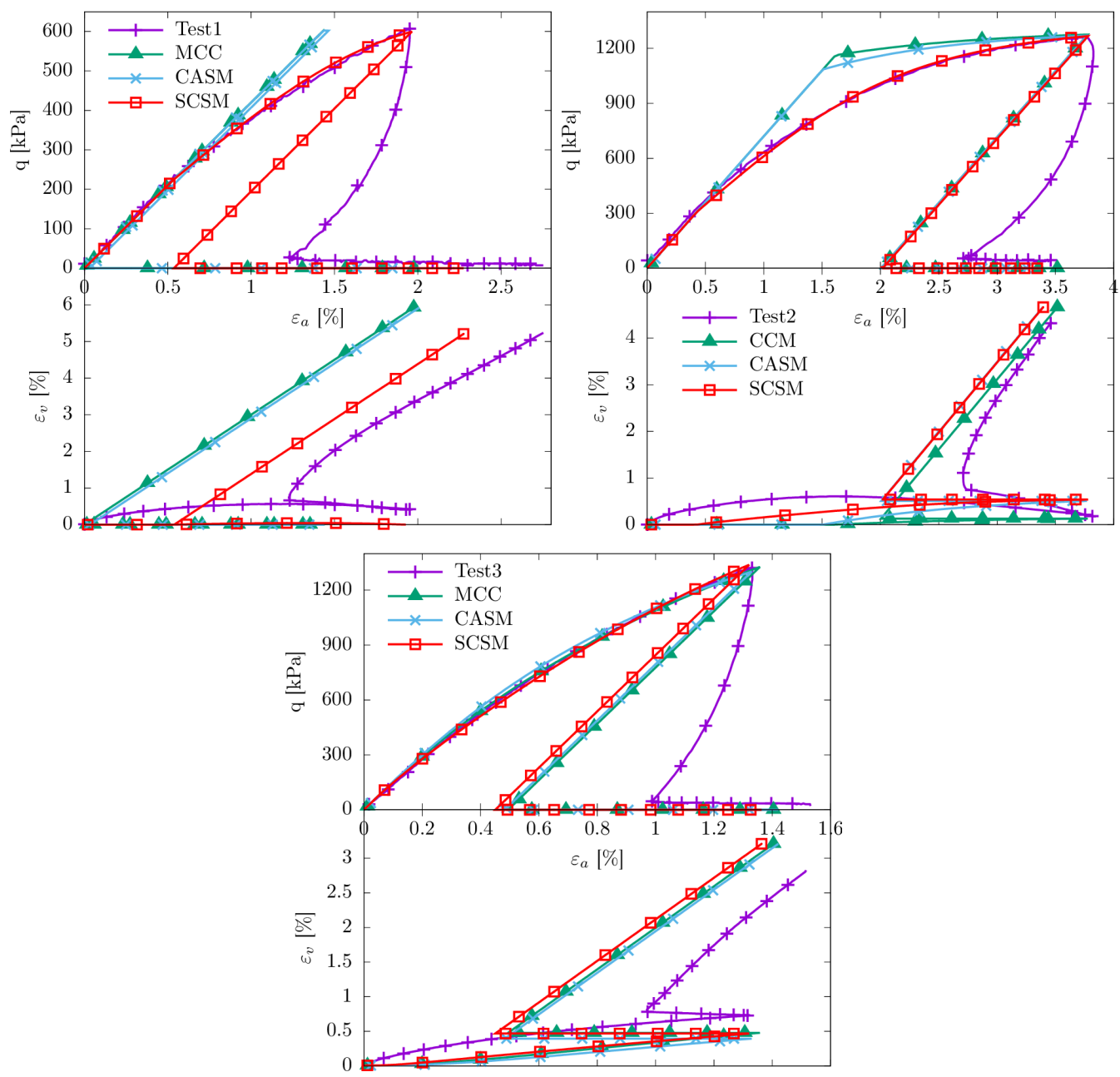

Figure 10: Simulation of specific stress controlled triaxial tests on a gray marl at different stress levels.

of destructuration and kinematic hard-ening were separately examined by the soil constitutive model presented by Amorosi, Boldini, and Germano (2008). These mechanisms could not help better simulate the nonlinear unloading.

Finally, the introduction of a stress-dependent stiffness different for un-loading was examined. In these stress-controlled tests, the effective mean pressure $p$ is constant and the deviator $q$ decreases in unloading whereas the confining pressure increases. The stiffness decays from the beginning of un-loading resulting in a nonlinear stress-strain response. A stress-dependent stiffness following the HSM elastic formulation (Schanz \& Vermeer, 2000) is then inappropriate in this case, as it increases with the confining pres-sure of a triaxial test. In this context, a simple exponential function, given by Equation 25 in its incremental form is proposed to simulate the nonlinear unloading. After examining the introduction of the mean pressure and the OCR in this equation, the use of the consolidation pressure $p c$ at the beginning of unloading gave the best fit.

$$
\dot{\gamma}=\frac{b}{p_{c}} \frac{\dot{q}}{\left(1+\frac{q}{p_{\text {ref }}}\right)^{c}}
$$

where $b$ and $c$ are dimensionless material parameters and $p_{\text {ref }}=100 \mathrm{kPa}$ is a normalizing reference pressure close to atmospheric pressure.

The fitting was performed using an error minimization procedure based on the least square method simultaneously on the three tests. The best unloading fit illustrated in Figure 11 was reached for $b=0.05$ and $c=$ 0.8 .

The overconsolidated tests are satisfactorily simulated contrary to the normally consolidated test which shows less non linearity than predicted with the proposed formulation. 


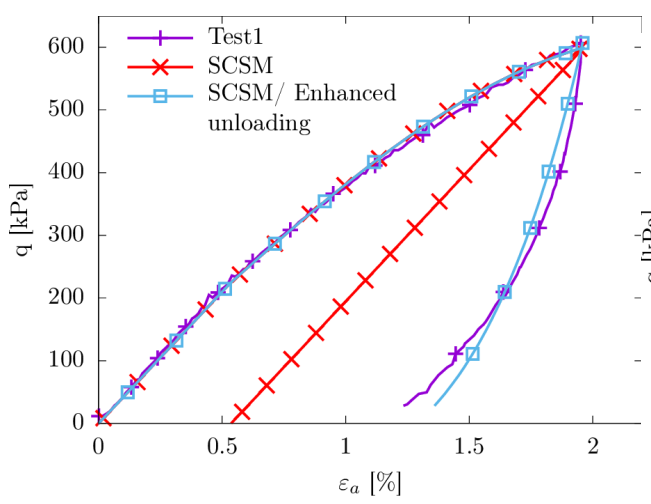

(a) Shear loading at $p=490 \mathrm{kPa}$

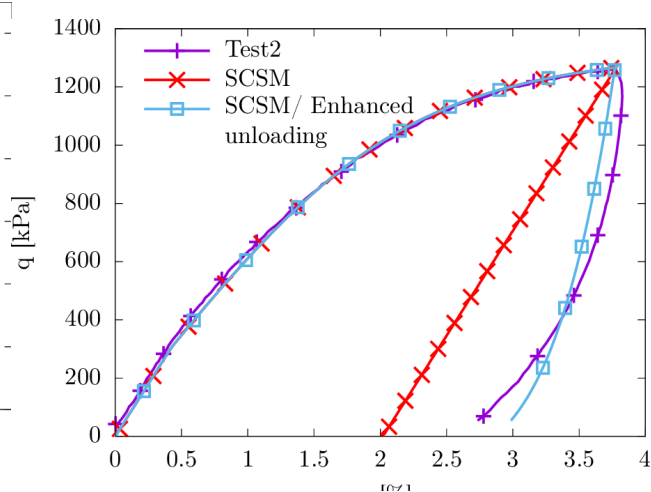

(b) Shear loading at $p=1000 \mathrm{kPa}$

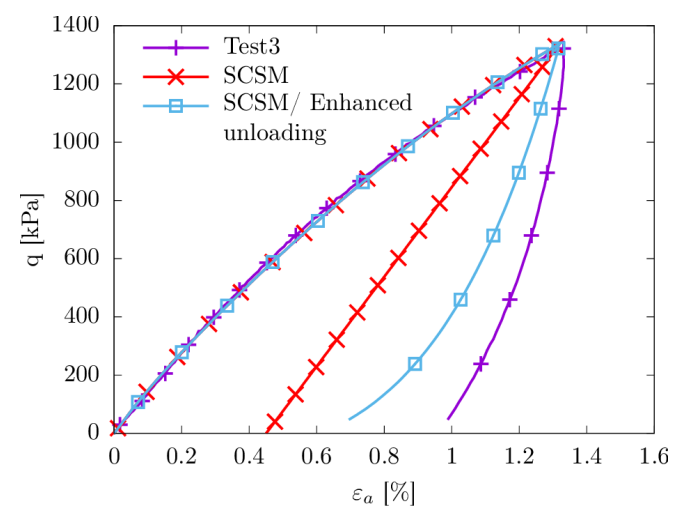

(c) Shear loading at $p=1800 \mathrm{kPa}$

Figure 11: Enhancement of the unloading simulation.

Further unloading tests at constant mean pressure should be carried out to enhance our comprehension of the mechanisms of unloading nonlinearity and to validate the proposed formulation.

\section{Conclusions and perspectives}

This paper presented an enhanced critical state model with deviatoric and volumetric hardening mechanisms. The proposed model was validated on undrained and drained classic triaxial tests on three clays at different consolidation states. The comparison with experimental data assessed the model's performance in successfully estimating the shear resistance of clay for both normally and overconsolidated states. In addition, triaxial tests with con-trolled stress paths were carried out for their ability to distinguish the impact of the shear hardening mechanism from that of volumetric hardening. The SCSM predictions agree fairly well with the experimental results in comparison to the CASM and MCC model that overestimate the shear resistance of OC clays and do not capture the non linearity of the stress-strain response.

Although the model's ability to capture the main characteristics of clayey soil behavior was satisfactory, further investigation and analysis will be required to improve the simulation of the unloading and increase its predictive capabilities.

Shear stress paths induced in some geotechnical projects have been the motivation behind the introduction of this soil constitutive model in the framework of critical state theory incorporating a deviatoric hardening mechanism in addition to the classic isotropic hardening one. The capacity of the SCSM to simulate purely deviatoric stress paths was demonstrated through specific triaxial tests. These tests present a new approach for the study of the relationship between deviatoric and isotropic hardening. The validation of SCSM performance on the structural level will be carried out in future work. 


\subsection{A Limit conditions}

Boundary conditions are only specified on boundary nodes. A question therefore arises when there is a relationship between applied conditions on many boundary points. For the sake of clarity, the problem can be stated with a limitation to two points without violation of the generalization.

Let us consider two points $x_{1}$ and $x_{2}$ on the boundary $\partial \Omega$ of a given domain $\Omega$ occupied by a continuum medium. The weak formulation can then be written as follows:

$$
\delta W=\ldots+F_{1} \delta U_{1}+F_{2} \delta U_{2}
$$

where $W$ is a symbolic quantity that involves a primary unknown $U$ and a secondary unknown $F . F_{1}$ and $F_{2}$ are given for Dirichlet boundary conditions, and $U_{1}$ and $U_{2}$ are given for Neumann conditions and a relationship between $F_{1}, F_{2}$ and $U_{1}$ and $U_{2}$ for Robin conditions. A problem is encountered when a relationship between $F_{1}$ and $F_{2}$ must be imposed. This case obviously goes beyond the classic classification of boundary conditions.

\subsection{Solution}

The relationship between $F_{1}$ and $F_{2}$ can always be reduced to a linear function in the rate form formulation. Thus, $F_{2}$ can be written as follows:

$$
F_{2}=a F_{1}+b
$$

Where $a$ and $b$ are known scalars. The unknown are $F_{1}$ and $F_{2}$.

By replacing in Equation 26, the following is derived:

$$
\delta W=\ldots+F_{1} \delta\left(U_{1}+a U_{2}\right)+b \delta U_{2}
$$

Equation 28 shows that $U_{1}+a U_{2}=V$ must be imposed since $F_{1}$ is unknown. Hence, equivalent boundary conditions for the definition of the problem are $F_{2}=b$ (for the Neumann boundary condition) and $U_{1}+a U_{2}=\mathrm{V}$ (for the Dirichlet boundary condition). The Lagrange multiplier related to the Dirichlet condition $U_{1}+a U_{2}=V$ is $F_{1}$ in the computing codes that uses Lagrange's method.

\subsection{Mechanical problem}

For the simulation of the specific test of pure shearing at constant mean pressure, we suppose that the soil or rock sample is a cylindrical specimen in a homogeneous stress and strain state. Using a cylindrical coordinate system, the vertical stress and deformation are respectively $F_{1}=\sigma_{z}$ and $U_{1}=\varepsilon_{z}$. Stress and strain in the perpendicular direction are respectively noted $U_{1}=\varepsilon_{\mathrm{r}}=\varepsilon_{\theta}$ and $F_{1}=\sigma_{\mathrm{r}^{\prime}}$. Performing a pure shear triaxial test at constant mean pressure $p$ consists of imposing

$$
\frac{\sigma_{z}+2 \sigma_{r}}{3}=\boldsymbol{p}
$$

used notations in the previous part, this means $a=-1$ and $b=-3 p$. This is equivalent to an imposed deformation $\varepsilon_{\mathrm{z}}-$ $\varepsilon_{\mathrm{r}}=\gamma$ and an imposed lateral pressure $\sigma_{r}=-\frac{3}{2} p$

\section{References}

[1] Amorosi, A., Boldini, D., \& Germano, V. (2008). Implicit integration of a mixed isotropic kinematic hardening plasticity model for structured clays. International journal for numerical and analytical methods in geomechanics, 32(10), 1173-1203.

[2] Barla, M. (1999). Tunnels in swelling ground: simulation of 3d stress paths by triaxial laboratory testing (Unpublished doctoral dissertation). Po-litecnico di Torino.

[3] Bishop, A. W., \& Henkel, D. J. (1957). The measurment of soil properties in the triaxial test. Edward Arnold Publishers.

[4] Chakraborty, T., Salgado, R., \& Loukidis, D. (2013). A twosurface plasticity model for clay. Computers and Geotechnics, 49, 170-190.

[5] Chen, J. (2017). A monotonic bounding surface critical state model for clays. Acta Geotechnica, 12, 225-230.

[6] Chen, Y., \& Yang, Z. (2017). A family of improved yield surfaces and their application in modeling of isotropically overconsolidated clays. Computers and Geotechnics, 90, 133-143.

[7] Dafalias, Y. F. (1986). An anisotropic critical state soil plasticity model. Mechanics Research communications, 13, 341-347.

[8] Dafalias, Y. F. (2016). Must critical state theory be revisited to include fabric effects? Acta Geotechnica, 11, 479-491.

[9] Dafalias, Y. F., Manzari, M. T., \& Papadimitriou, A. G. (2006). Saniclay: simple anisotropic clay plasticity model. International Journal For Numerical and Analytical Methods In Geomechanics, 30, 1231-1257.

[10] Dafalias, Y. F., \& Taiebat, M. (2013). Anatomy of rotational hardening in clay plasticity. Géotechnique, 63, 1406-1418.

[11] DAO, L. Q. (2015). Etude du comportement anisotrope de l'argile de boom (Unpublished doctoral dissertation). Ecole des Ponts ParisTech.

[12] Desai, C., Somasundaram, S., \& Frantziskonis, G. (1986). A hierarchical ap-proach for constitutive modelling of geologic materials. International Journal for Numerical and Analytical Methods in Geomechanics, 10(3), 225-257.

[13] Desai, C. S. (1980). A general basis for yield, failure and potential func-tions in plasticity. International Journal for 
Numerical and Analytical Methods in Geomechanics, 4(4), 361-375.

[14] Einav, I., \& Puzrin, A. M. (2004). Pressure-dependent elasticity and energy conservation in elastoplastic models for soils. Journal of Geotechnical and Geoenvironmental engineering, 63(8), 81-92.

[15] Gasparre, A. (2005). Advanced laboratory characterization of london clay (Unpublished doctoral dissertation). Imperial College London.

[16] Gens, A., \& Potts, D. M. (1988). Critical state models in computational geomechanics. Engineering Computation, 5, 178-197.

[17] Gilelron, N. (2016). Use of the hardening soil model for urban tunnels design. In 25th european young geotechnical engineers conference, sibiu, romania.

[18] Gilleron, N., \& Bourgeois, E. (2016). Influence of deviatoric stress depen-dent stiffness on settlement trough width in $2 \mathrm{~d}$ and 3d finite element modelling of tunnelling. In (p. 567-576).

[19] Hattab, M., \& Hicher, P.-Y. (2004). Dilating behaviour of overconsolidated clay. Soils and Foundations, 44(4), 27-40.

[20] Hong, P. Y., Pereira, J. M., Tang, A. M., \& Cui, Y. J. (2016). A two-surface plasticity model for stiff clay. Acta Geotechnica, 11, 871-885.

[21] Houlbsy, G. T., Amorosi, A., \& Rojas, E. (2005). Elastic moduli of soils dependent on pressure: a hyperelastic formulation. Géotechnique, 55(5), 383-392.

[22] Jin, Z. Y., Xu, Q., \& Hicher, P. Y. (2017). Estimation of critical state-related formula in advanced constitutive modeling of granular mate-rial. Acta Geotechnica, 12(6), 1329-1351.

[23] Lagioia, R., \& Potts, D. M. (1988). A new versatile expression for yield and plastic potential surfaces. Computers and Geotechnics, 5, 178-197.

[24] Liu, M., \& Carter, J. (2002). A structured cam clay model. Canadian Geotechnical Journal, 39, 1313-1332.

[25] Mair, R. J. (1979). Centrifugal modelling of tunnel construction in sof clay (Unpublished doctoral dissertation). Cambridge University.

[26] Mroz, Z., \& Zienkiewicz, O. C. (1984). Uniform formulation of constitutive equations for clays and sands. In Mechanics of engineering materials (John Wiley and Sons ed., p. 415-449).

[27] Obrzud, F. (2010). On the use of the hardening soil small strain model in geotechnical practice. Numerics in Geotechnics and Structures.

[28] Panet, M. (1995). Calcul des tunnels par la méthode convergence-confinement. Presses de l'Ecole Nationale des Ponts et Chaussées.

[29] Potts, D. M., \& Zdravkovic, L. (1999). Finite element analysis in geotechnical engineering : theory. Thomas Telford.

[30] Roscoe, K. H., \& Burland, J. B. (1968). On the generalized stress-strain behevior of wet clay. Cambridge University Press.

[31] Roscoe, K. H., Schofield, A. N., \& Wroth, C. P. (1958). On the yielding of soils. Géotechnique, 8, 22-52.

[32] Schanz, T., \& Vermeer, P. (2000). The hardening soil model: Formulation and verification. Beyond 2000 in Computational Geotechnics.

[33] Schofield, A., \& Wroth, P. (1968). Critical state soil mechanics (Vol. 310). McGraw-Hill London.

[34] Serratrice, J. F. (2002). Outils et procédures de caractérisation des sols indurés et des roches tendres : l'expérience du lrpc d'aix en provence. PARAM, 313-326.
[35] Suebsuk, J., Horpibulsuk, S., \& Liu, M. D. (2010). Modified structured cam clay: A generalised critical state model for destructured, nat-urally structured and artificially structured clays. Computers and Geotechnics, 37, 956-968.

[36] Suebsuk, J., Horpibulsuk, S., \& Liu, M. D. (2011). A critical state soil model for overconsolidated clays. Computers and Geotechnics, 38, 648-658.

[37] Sultan, N., Cui, Y.-J., \& Delage, P. (2010). Yielding and plastic behaviour of boom clay. Géotechnique, 60(9), 657-666.

[38] Tijani, M. (1996). Short description of viplef code. In Coupled thermo-hydro-mechanical processes of fractured media: mathematical and experimental studies (Elsevier ed., p. 507 511).

[39] Tijani, M. (2008). Contribution à l'étude thermomécanique des cavités réalisées par lessivage dans des formations géologiques salines. Uni-versité Pierre et Marie Curie.

[40] Truty, A., \& Obrzud, R. (2015). Improved formulation of the hardening soil model in the context of modeling the undrained behavior of cohesive soils. Studia Geotechnica et Mechanica, 37(2), 61-68.

[41] Wood, D. M. (2003). Geotechnical modelling. CRC Press.

[42] Yu, H. S. (1998). Casm: A unified state parameter model for clay and sand. International Journal For Numerical and Analytical Methods In Geomechanics, 22, 1621-653.

[43] Yu, H. S. (2006). Plasticity and geotechnics. Springer.

[44] Zytynski, M., Randolph, M. F., \& Wroth, C. P. (1978). On modelling the unloading-reloading behaviour of soils. International Journal for Numerical and Analytical Methods in Geomechanics, 2, 87-94. 